AperTO - Archivio Istituzionale Open Access dell'Università di Torino

Stopping movements: when others slow us down

This is a pre print version of the following article:

Original Citation:

Availability:

This version is available http://hdl.handle.net/2318/148290

since

Published version:

DOI:10.1111/ejn.12645

Terms of use:

Open Access

Anyone can freely access the full text of works made available as "Open Access". Works made available under a Creative Commons license can be used according to the terms and conditions of said license. Use of all other works requires consent of the right holder (author or publisher) if not exempted from copyright protection by the applicable law. 


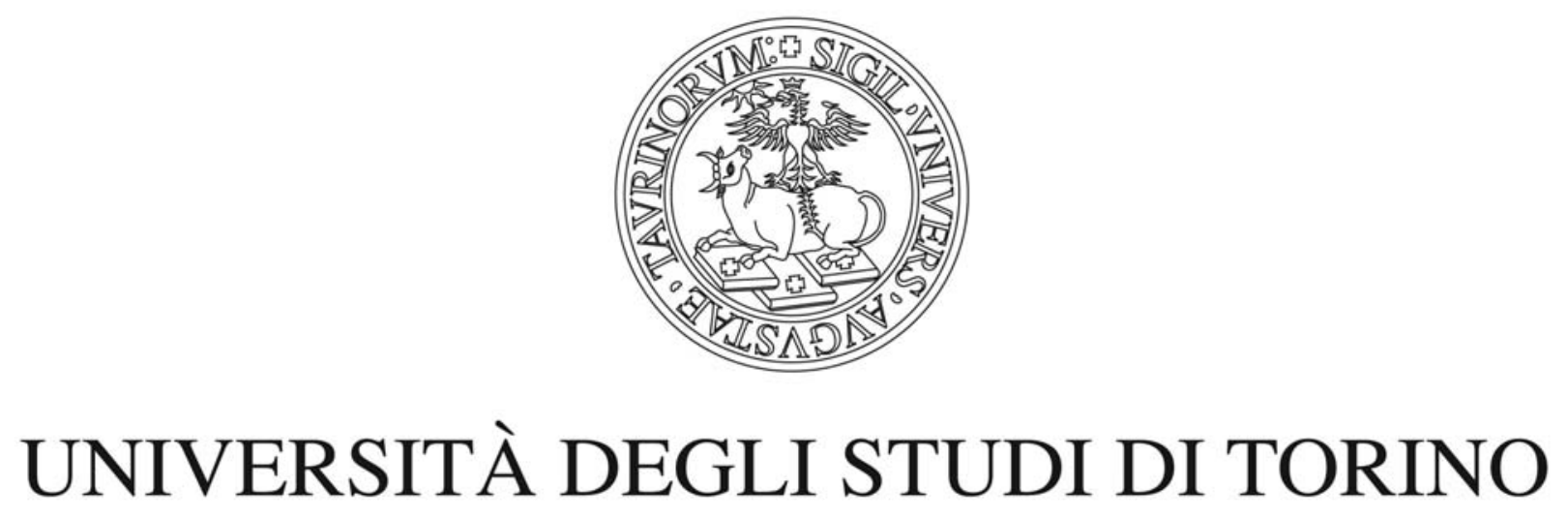

This is an author version of the contribution published on:

Questa è la versione dell'autore dell'opera:

Cavallo A., Catmur C., Sowden S., lanì F., Becchio C. (2014). Stopping movements: when others slow us down. European Journal of Neuroscience, 40, 2842-2849.

This article may not exactly replicate the final version published in the journal. It is not the copy of record.

The definitive version is available at:

La versione definitiva è disponibile alla URL:

http://onlinelibrary.wiley.com/doi/10.1111/ejn.12645/full 
Journal section: Research reports

\section{Stopping movements: when others slow us down}

Andrea Cavallo ${ }^{1}$, Caroline Catmur ${ }^{2}$, Sophie Sowden ${ }^{2,3}$, Francesco Ianì ${ }^{1}$, Cristina Becchio ${ }^{4,1^{*}}$

${ }^{1}$ Università di Torino, Dipartimento di Psicologia, Centro di Scienza Cognitiva, Turin, Italy

${ }^{2}$ Department of Psychology, University of Surrey, Guildford, GU2 7XH, UK

${ }^{3}$ MRC Social, Genetic \& Developmental Psychiatry Centre, Institute of Psychiatry, King's College London, London, SE5 8AF, UK

${ }^{4}$ Department of Robotics, Brain, and Cognitive Science, Italian Institute of Technology, Genova, Italy

* correspondence to: Cristina Becchio, Department of Psychology, University of Turin; Via Po, 14, 10123 Turin, Italy; Tel: +39 011 6703072; Fax: +39 011 8146231; E-mail address:

cristina.becchio@unito.it

Running title: Stopping movements

Page counts: 26; Figure counts: 2; Table counts: 2

Word counts: i) whole manuscript: 5711; ii) abstract: 120; iii) introduction: 949

Keywords: Joint stopping, action co-representation, transcranial magnetic stimulation, motor evoked potentials 


\begin{abstract}
Previous research has shown that performing joint actions can lead to the representation of both one's own and others' actions. In the present study we explored the influence of co-representation on response stopping. Are joint actions more difficult to stop than solo actions? Using a variation of the stop-signal task, we found that participants needed more time to stop a planned joint action compared to a planned solo action (Experiment 1). This effect was not observed when participants performed the task in the presence of a passive observer (Experiment 2). A third transcranial magnetic stimulation experiment (Experiment 3) demonstrated that joint stopping recruited a more selective suppression mechanism than solo stopping. Taken together these results suggest that participants used a global inhibition mechanism when acting alone; however, they recruited a more selective and slower suppression mechanism when acting with someone else.
\end{abstract}




\section{Introduction}

Many every-day activities require the ability to efficiently adjust and coordinate actions with others to achieve a common goal (Sebanz et al., 2006). Lifting a table up a narrow staircase, singing a duet or shaking hands are prime examples of what can be referred to as shared cooperative activities (Bratman, 1992) or joint actions (Sebanz et al., 2006).

Previous research has shown that performing joint actions can lead to the representation of both one's own and others' actions. This occurs, for example, when the two stimulus-response mappings of a two-choice task are distributed between two actors. Even when there is no need to take the other's mapping into account to perform the instructed task, a response conflict is observed when a task-irrelevant aspect of a stimulus activates a response corresponding to the task-relevant response of the other actor (Sebanz et al., 2003). In line with ideomotor theories (Greenwald, 1970; Jeannerod \& Frak, 1999; Prinz, 1997), this has been taken to suggest that in planning joint actions, participants form task representations that specify not only their own part, but also the part to be performed by the co-actor (Sebanz et al., 2003; Sebanz et al., 2005). The action alternatives at the other's disposal might become represented in a functionally similar way as their own and have a specific impact on their own acting (for a review see Sebanz et al., 2006).

Co-representation of others' actions during coordinated planning has been shown to influence stimulus processing (Heed et al., 2010), action monitoring (Schuch \& Tipper, 2007), control (Sebanz et al., 2006; Tsai et al., 2006), and prediction processes during the ensuing interaction (Ramnani \& Miall, 2004; for review see Knoblich et al., 2011). A far less explored issue is whether co-representation also influences task performance when people are asked to stop rather than to perform a joint action.

Joint action inhibition: the stop-signal paradigm 
A useful tool for studying the processes involved in stopping a motor response is the stopsignal paradigm (Lappin \& Eriksen, 1966), a choice reaction time (RT) task which asks participants to occasionally withhold their ongoing response when a stop-signal appears. Performance in the stop-signal paradigm is modelled as a race between a 'go process', triggered by the presentation of the Go stimulus, and a 'stop process', triggered by the presentation of the Stop stimulus (Logan, 1981; Logan \& Cowan, 1984; for a review see Verbruggen \& Logan, 2009). According to the race model, only when the cognitive process triggered by the Stop signal terminates before the end of the 'go' cognitive process, can participants correctly suppress their movement. By constantly modifying the latency between the Go signal and the Stop signal in a way that the overall movement prevention probability is approximately $50 \%$, the latency of the stop process (stop-signal reaction time; SSRT) can be estimated (Lappin \& Eriksen, 1966; Ollman, 1973; Logan, 1981). Here we adapted the stop-signal paradigm to determine whether and how performing the task with someone else influences the stopping processes. In three experiments, participants performed the stop-signal task alone (Solo condition) and alongside a co-actor (Joint condition) or a passive observer. In Experiment 1, we used a variation of the stop-signal task to behaviourally dissociate solo and joint stopping. In Experiment 2 we tested whether the dissociation between solo and joint stopping might be explained by the mere presence versus absence of a person sitting next to the participant. In Experiment 3, using transcranial magnetic stimulation (TMS), we probed whether solo stopping and joint stopping recruit different neural mechanisms of movement suppression. We tested our results against three hypotheses.

No effect on stopping. First, as the co-actor's task was irrelevant (see below), the co-actor's actions may be not represented at all and may therefore exert no influence on the participant's performance. If so, performance should be the same in the Solo and in the Joint conditions.

Non-specific effect of social facilitation. Second, in line with social facilitation, the mere presence of a co-actor may exert a general effect on task performance (e.g., Aiello \& Douthitt, 2001). It has been shown, for example, that when participants are engaged in a dialogue, social 
facilitation can increase alertness and counter the effects of sleep deprivation (Bard et al., 1996).

Social facilitation effects are not moderated by the specific actions carried out by others. Rather, the mere presence of others often leads to similar effects as when a group of individuals engage in the same actions (Bond \& Titus, 1983). Under this account, a non-specific effect of the other's presence should thus be expected.

Specific effect of action co-representation. Finally, in line with the joint action literature, it is possible that the participants represent the other's specific task demands, even when the co-actor's task is irrelevant (Sebanz et al., 2003; Sebanz et al., 2005). Such representation would create an additional task set in which the stimuli are associated with the co-actor's responses: that is, the Go stimulus would now activate representations of both the participant's responses and those of the coactor. In this case, on stop-signal trials, participants may require a more selective mechanism to control their own responses independently of those of the co-actor (Aron, 2011). As selective stopping has been shown to be slower than global stopping (e.g. Claffey et al., 2010; Coxon et al., 2007; Majid et al., 2012; for a review see Stinear et al., 2009), one would expect that the time which participants require to stop their response is lengthened in the joint condition compared to the individual condition. In line with this account, the distinctive finding would thus be that stopping is more selective and also slower when performing the task alongside a co-actor than when performing the task alone.

\section{Experiment 1}

In Experiment 1 we used the stop-signal paradigm to behaviourally dissociate solo stopping and joint stopping. Participants were asked to perform a choice RT task alone (Solo condition) and alongside a co-actor (Joint condition). In this task they had to respond to a target (i.e. press either a left or a right key depending on the stimulus shape) as quickly as possible. Occasionally, the target stimulus was followed by an auditory tone which served as a Stop signal. We measured the speed with which participants stopped their response, i.e., the stop signal reaction time (SSRT). SSRT 
represents the time interval between the start and the end of the stopping process and can be estimated on the basis of the RT distribution observed on no-signal trials (Go RT) and the probability of responding during stop-signal trials observed for a given stop-signal delay (SSD) (for a review, see Logan, 1994). If the presence of the co-actor facilitates performance in a general way, then we would expect a general speeding up of both go and stop processes. In contrast, if corepresentation specifically influences stopping, then we would expect SSRT to be longer in the Joint condition than in the Solo condition.

\section{Material and Methods}

Participants. Twelve healthy participants ( 8 female; aged $21-34$ years, mean age $=25.58$ ) with no history of neurological or hearing problems took part in the experiment. They were all right-handed and had normal or corrected-to-normal vision. Before the experimental session each participant was naïve as to the purposes of the study and signed an informed consent; information about the experimental hypothesis was given only at the end of the experiment. The experimental procedures for both experiments were approved by the local Ethics Committee and were carried out in accordance with the principles of the revised Helsinki Declaration (World Medical Associations General Assembly, 2008).

Task and Procedure. Participants performed a standard stop-signal task (based on Verbruggen et al., 2008). Stimuli were presented on a 17-inch monitor ( 1600 x 900 pixels, refresh frequency, $60 \mathrm{~Hz})$ at a viewing distance of $70 \mathrm{~cm}$. Participants were seated in a comfortable chair, with their left and right index fingers on the ' $A$ ' and ' $L$ ' keys of a standard keyboard. Each participant performed the stop-signal task alone (Solo condition) and alongside a female experimenter acting as a co-actor (Joint condition). In the Joint condition the participant and the co-actor sat side-by-side in front of the monitor with a distance of about $40 \mathrm{~cm}$ between them. In the Solo condition, the chair next to the participant remained empty. The monitor was equidistant from the two chairs. Task and 
procedure were identical in the Solo and in the Joint conditions, except that in the Joint condition instructions required both agents to perform the task. The co-actor was introduced to the participant as another participant. The participant was told that the co-actor would complete the solo part of the experiment at another time. Participants were not required to pay attention to or to coordinate their actions with those of the co-actor.

Each trial began with a white fixation cross lasting $250 \mathrm{~ms}$, followed by the imperative Go signal. The Go signal was either a white circle or square. Participants were instructed to press the 'A' key for the circle, and the ' $L$ ' key for the square. They were asked to respond to the Go signal as fast and accurately as possible. Stimuli remained on the screen until a response was made, or until $1250 \mathrm{~ms}$ had elapsed. In $25 \%$ of trials (Stop trials), the imperative Go signal was suddenly followed by an auditory Stop signal $(750 \mathrm{~Hz}, 75 \mathrm{~ms}), 24$ times for the circle and 24 times for the square, instructing participants to withhold their responses. The Stop signal was presented after a variable delay (SSD) initially set at $250 \mathrm{~ms}$. The SSD was adjusted continuously with the staircase converging tracking procedure (Band et al., 2003): when stopping succeeded, SSD increased by 50 ms; when stopping failed, SSD decreased by $50 \mathrm{~ms}$. This tracking procedure yields a probability of .50 of stopping to a stop signal $[p($ stop $\mid$ signal $) ; p(\mathrm{~S} \mid \mathrm{S})]$ (Levitt, 1971). In both the Solo and the Joint condition the tracking procedure was based on the actual participant responses so that the SSD was exclusively adapted to follow the participant's performance. The SSRT was calculated using the integration method: for each participant, each SSD was subtracted from the $n$th RT, where $n$ is the number of RTs in the RT distribution multiplied by the overall probability of responding at a given delay $[p($ respond $\mid$ signal $) ; p(\mathrm{R} \mid \mathrm{S})]$. Thus, SSRT was estimated for every SSD and was then averaged across SSDs. (Verbruggen et al., 2013; Verbruggen \& Logan, 2009). The intertrial interval (ITI) lasted 2000 ms. Each condition (Solo and Joint) consisted of 192 randomly presented trials (144 Go trials and 48 Stop trials) equally distributed across four mini blocks of 48 trials each. Before each condition, participants completed a practice phase, in which they were presented with 32 trials (24 
Go and 8 Stop). The order of the Solo and Joint conditions was counter-balanced across participants. The experiment lasted approximately 25 minutes.

Data analysis. The accuracy of Go trials, the mean RT of correct Go trials (Go RT), the probability of stopping $[\mathrm{p}(\mathrm{S} \mid \mathrm{S})]$, the mean SSD, and the SSRT were calculated separately for both the Solo and Joint conditions. One sample t-tests revealed that the $\mathrm{p}(\mathrm{S} \mid \mathrm{S})$ was not significantly different from .50 in both the Solo $\left(t_{11}=-0.966, p=.355\right)$ and the Joint $\left(t_{11}=-1.401, p=.189\right)$ conditions, indicating that the staircase converging tracking procedure was successful.

Paired t-tests (2-tailed) on the above indices were performed to ascertain whether there were significant differences between the Solo and Joint conditions.

\section{Results}

Table 1 summarizes the key measures for both conditions. Participants required more time to stop an initiated response when they performed the task alongside another person. The SSRT was significantly longer $\left(t_{11}=-2.470, p=.031\right)$ in the Joint condition compared to the Solo condition. Similarly, the SSD was significantly shorter in the Joint condition compared to the Solo condition $\left(t_{11}=-2.996, p=.012\right)$. In contrast, no difference in Go RTs was observed between the Solo and Joint conditions $\left(\mathrm{t}_{11}=0.722, \mathrm{p}=.457\right)$. Relative to our predictions, this finding of a longer latency of the stopping process in the Joint condition compared to the Solo condition is compatible with the hypothesis that in the Joint condition, participants represented the other's responses as well as their own, and were thus induced to use a more selective, but slower mechanism to stop their own response.

\section{Experiment 2}


A potentially relevant difference between the Joint condition and Solo condition was the presence versus absence of a person sitting next to the participant. This difference, rather than action co-representation, could be the source of the longer SSRT obtained in Experiment 1. To rule out this possibility, in Experiment 2 we asked participants to perform the stop-signal paradigm alone or in the presence of a passive observer. If the mere presence of another person is responsible for the effect, then a longer SSRT should also be obtained when the passive observer was seated next to the participant. In contrast, if stopping is specifically influenced by the co-actor's responses, then no effect on the stopping process should be expected.

\section{Material and Methods}

Participants. Twelve new healthy participants ( 7 female; aged $20-27$ years, mean age $=23.17$ ) with no history of neurological or hearing problems took part in the experiment. They were all righthanded and had normal or corrected-to-normal vision. Participants were naïve as to the purpose of the study and signed an informed consent; information about the experimental hypothesis was given only at the end of the experiment.

Task, Procedure and Data Analysis. Task, procedure, and data analysis were the same as those used in Experiment 1 except that here participants performed the stop-signal task alone (Solo condition) and alongside a passive observer (Passive observer condition) who sat beside them without acting. As in Experiment 1, participants were told that the co-actor would complete the Solo part of the experiment at another time.

\section{Results}


One sample t-tests revealed that the $\mathrm{p}(\mathrm{S} \mid \mathrm{S})$ was not significantly different from .50 in both the Solo $\left(t_{11}=0.581, p=.573\right)$ and the Passive observer $\left(t_{11}=1.239, p=.241\right)$ conditions, indicating that the staircase converging tracking procedure was successful.

Paired t-tests (2-tailed) on the Go RT $\left(t_{11}=0.234, p=.819\right)$, the SSRT $\left(t_{11}=0.155, p=.879\right)$, and the SSD $\left(t_{11}=0.050, p=.961\right)$ revealed no differences between the Solo and the Passive observer conditions (Table S1). This indicates that the mere presence vs. absence of a person sitting next to the participant was not sufficient to influence task performance.

\section{Experiment 3}

The finding of a longer latency of the stopping process when participants performed the task alongside a co-actor (Experiment 1), but not when they performed the task in the presence of a passive observer (Experiment 2) is compatible with the hypothesis that in the Joint condition, participants represented the other's responses as well as their own, and were thus induced to use a more selective, but slower mechanism to stop their own response. However, differences in SSRT do not prove on their own that a more selective mechanism was engaged during joint stopping (Aron \& Verbruggen, 2008). To obtain a direct measure of the selectivity of the joint stopping mechanism, in a third TMS experiment, we measured the corticospinal modulation of a task-irrelevant muscle during the stop-signal task.

When a global mechanism is used to stop the hand, 'widespread pulses' inhibit the motor system generally and a significant decrease in excitability is observed not only in the hand muscle, but also in task-irrelevant effectors (Badry et al., 2009). Consistent with this, Majid and colleagues (2012) demonstrated that for standard, non-selective stopping, stopping the hand led to suppression of a task-irrelevant leg muscle. This diffuse suppression effect was not observed when participants were required to stop selectively, i.e., when they were cued in advance about which response to stop. 
Following the same logic, in Experiment 3 we used TMS to probe the corticospinal excitability of a task-irrelevant leg muscle while participants performed a stop-signal task alone or alongside a co-actor. We treated task-irrelevant leg suppression as a 'TMS signature' of global versus selective stopping. We predicted that if joint stopping engages a more selective mechanism, then stopping should be slower and leg suppression reduced (i.e. corticospinal excitability should be greater) for the Joint compared to the Solo condition. Such a result would corroborate the hypothesis that a different suppression mechanism is recruited for jointly performed actions relative to individually performed actions.

\section{Materials and Methods}

Participants. Twenty-one new healthy volunteers (16 female) aged 18-31 (mean 21.8) took part in Experiment 3. All were right-handed, had normal or corrected-to-normal visual acuity and were free from any contraindication to TMS (Wassermann, 1998; Rossi, Hallett, Rossini, \& Pascual-Leone, 2009). Before the experimental session each participant was naïve as to the purposes of the study and signed an informed consent; information about the experimental hypothesis was given only at the end of the experiment. Participants were financially compensated for their time. None experienced discomfort during TMS.

Task and Procedure. Participants performed a TMS-adapted version (based on Majid et al., 2012) of the standard stop-signal task. Stimuli were presented on a 19-inch monitor (resolution $1280 \mathrm{x}$ 1024 pixels, refresh frequency, $60 \mathrm{~Hz}$ ) at a viewing distance of $90 \mathrm{~cm}$. A chinrest was used to provide support and minimise head movements during TMS. Each trial began with a 500 ms yellow fixation cross, followed by a $500 \mathrm{~ms}$ blank screen. The imperative Go signal (circle: 'A' response; square: ' $L$ ' response) was then presented. On Go trials ( $67 \%$ of all trials), the Go signal remained 
on the screen until a response was made, or until $900 \mathrm{~ms}$ had elapsed (Figure 1A). On Stop trials (33\% of all trials) a red cross Stop signal appeared 35 times over the circle and 35 times over the square indicating that the participant and co-actor should withhold their responses. The Stop signal remained on the monitor until the end of the trial (Figure 1B). As in Experiment 1 and 2, the SSD was initially set at $250 \mathrm{~ms}$ and was then adjusted continuously with the staircase converging tracking procedure (Band et al., 2003). The SSD was calculated based on the participant's responses. The ITI ranged from 4 to $6 \mathrm{~s}$ (mean $5 \mathrm{~s}$ ). The experimental procedure was divided into 3 sessions: i) 'Practice' without TMS; ii) 'Solo' condition; and iii) 'Joint' condition. For each participant, in order to calculate the timing of the TMS pulse, the experiment started with the Practice session (66 trials; 44 Go and 22 Stop) in which the co-actor was not present. After the Practice, the Solo and the Joint conditions (each including two blocks of 105 trials; 70 Go and 35 Stop) were run in a counterbalanced order across participants. TMS pulses were delivered on 90 out of 105 trials in each block (60 Go trials and 30 Stop trials) during the response period calculated by subtracting $100 \mathrm{~ms}$ from mean Go RT obtained during the Practice session (Training Go RT - 100 ms; Badry et al., 2009; Majid et al., 2012). 15 additional motor evoked potentials (MEPs) were recorded $300 \mathrm{~ms}$ before the onset of the Go signal for 10 Go trials and 5 Stop trials and served as a baseline for the response period MEPs. The entire experimental procedure (Practice, two Solo blocks, two Joint blocks) lasted approximately 100 minutes. Stimulus-presentation timing, EMG recording and TMS triggering, as well as stimulus randomisation, were controlled by E-Prime V2.0 Software (Psychology Software Tools Inc., Pittsburgh, PA, USA) running on a PC.

Electromyographic and TMS Recording. TMS pulses were administered using a Magstim Rapid ${ }^{2}$ stimulator (Magstim, Whitlan, Dyfed, Wales, UK) connected to a $70 \mathrm{~mm}$ figure-of-eight coil initially positioned $2 \mathrm{~cm}$ anterior to the vertex of the head of the participant $(\mathrm{Cz}$; the point half-way between the nasion and the inion) approximately corresponding to the midline primary motor cortex (M1) representations of the Tibialis Anterior (TA) muscles. During TMS preparation, the coil was 
positioned in correspondence with the optimal scalp position (OSP), defined as the position from which MEPs with maximal amplitude were recorded in either of the 2 TA muscles (Majid et al., 2012). To find the individual OSP, the coil was moved in steps of $0.5 \mathrm{~cm}$ over the motor cortex and the OSP was marked on a bathing cap worn by participants. To increase the likelihood of finding the OSP, participants were requested to slightly activate their TA muscles by placing their heels on the floor and the toes upon a low-rise step. Once the OSP was found, the individual resting motor threshold (rMT) was determined as the lowest stimulus intensity able to generate MEPs (no less than $50 \mu \mathrm{V}$ peak-to-peak amplitude) in five out of ten consecutive TMS pulses (Rossini et al. 1994). Five participants were excluded due to difficulty in finding either individual OSP or rMT. During the recording sessions, stimulation intensity was $115 \%$ of the rMT and it ranged from $53 \%$ to $85 \%$ (mean 76.3\%) of the maximum stimulator intensity. The OSP was located over the right motor cortex (stimulating the left leg) in 13 participants; in 3 participants it was located over the left motor cortex (stimulating the right leg). MEPs were recorded from the TA muscles (muscles responsible for the feet dorsoflexion) through pairs of $\mathrm{Ag}-\mathrm{AgCl}$ surface electrodes (10 mm diameter) placed over the muscle belly (active electrode) and over the associated joint or tendon (reference electrode) in a classical belly-tendon montage. Electrodes were connected to an isolated portable ExG input box linked to the main EMG amplifier for signal transmission via a twin fiber optic cable (Professional BrainAmp ExG MR, Brain Products, Munich, Germany). The ground electrodes were placed over the participants' lateral malleoli of both legs and were connected to the common input of the ExG input box. A notch filter $(50 \mathrm{~Hz})$ was used and responses were sampled $(5000 \mathrm{~Hz})$, amplified, band-pass filtered $(3 \mathrm{~Hz}-3000 \mathrm{~Hz})$, and stored on a PC for off-line analysis. A prestimulus recording of $100 \mathrm{~ms}$ was used to check for the presence of EMG activity before the TMS pulse. In order to prevent contamination of MEP measurements by background EMG activity, trials with any background activity greater than $100 \mu \mathrm{V}$ in the $100 \mathrm{~ms}$ window preceding the TMS pulse were excluded from the MEP analysis (e.g. Duque \& Ivry 2009; Cavallo et al., 2012). EMG data were collected for $200 \mathrm{~ms}$ after the TMS pulse. 
Data analysis. EMG data were analyzed off-line using Brain Vision Analyzer software (Brain Products GmbH, Munich, Germany). Background EMG level prior to TMS was checked for each trial. Individual mean peak-to-peak amplitudes of MEPs recorded from TA muscles were calculated separately for Go and Stop trials. MEP amplitudes deviating more than 2 standard deviations (SD) from the mean of each experimental condition $(<2 \%)$, single trials contaminated by muscular preactivation $(<2 \%)$ and Stop trials in which the TMS trigger was delivered before the Stop signal (41 out of 1920 trials) were excluded from the analysis. Additionally, to ensure that any effect on MEP amplitude was not due to differences in background EMG level prior to TMS, the root mean square (rms) EMG obtained from the $100 \mathrm{~ms}$ window before the TMS pulse was calculated. A repeated measure 2 (Solo, Joint) x 2 (Go, Stop) ANOVA was then performed. Neither main effects $[$ Condition $\mathrm{p}=.775(\mathrm{rms}$ Solo $=4.49 ; \mathrm{rms}$ Joint $=4.32) ;$ Trial type $\mathrm{p}=.289(\mathrm{rms}$ Go $=4.26 ; \mathrm{rms}$ Stop $=4.54)$ ] nor an interaction (Condition $x$ Trial type, $p=.242$ ) were observed in the rms data (see Figure S1 for a representation of rms prior to TMS pulse).

To allow unbiased comparison between sessions, for each participant, MEP amplitudes were converted into a proportion of the baseline value. To simplify data presentation and allow comparison across conditions, Stop Ratios, as shown below, were calculated for each condition by dividing the baseline normalized mean MEP amplitude of the Stop trials by the mean MEP amplitude resulting from the Go trials.

$$
\text { Stop ratio }=\frac{\text { MEP Stop trials }}{\text { MEP Go trials }}
$$

The lower the value of this index, the stronger the task-irrelevant leg suppression.

To compare the MEP Stop Ratios for the Solo and the Joint conditions, a paired-sample ttest (2-tailed) was performed. 


\section{Results}

Table 2 summarizes the key measures computed for both the Solo and the Joint conditions. As in Experiment 1, SSRT was prolonged $\left(t_{15}=-2.369, p=.032\right)$ for the Joint condition compared to the Solo condition. Consistent with our prediction, during Stop trials leg suppression was reduced $\left(t_{15}=\right.$ -2.372, $p=.032$ ) for the Joint relative to the Solo condition (Figure 2). Critically, no difference in Go MEPs was observed $\left(\mathrm{t}_{15}=.389 ; \mathrm{p}=.352\right.$, one tailed), therefore excluding the possibility that differences in Stop Ratios may arise from larger Go MEP amplitudes in the Solo than Joint condition.

Taken together, behavioural and TMS data suggest that, compared to the Solo condition, participants employed a more selective and slower suppression mechanism when responding alongside a co-actor.

\section{Discussion}

The present stop-signal study was designed to test the hypothesis that joint context modulates stopping processes. Performing joint actions can lead to the representation of both one's own and others' actions (e.g., Sebanz et al., 2003). We reasoned that if participants represent the stimulusresponse mappings of the co-actor alongside their own, then they may require a more selective but also slower mechanism to control their own responses when performing the task alongside a coactor. Under this account, stopping should thus be more selective and slower in the Joint condition compared to the Solo condition. Experiment 1 demonstrated that the latency of the stop process (SSRT) was indeed prolonged in the Joint condition relative to the Solo condition. This effect was not observed when participants performed the task in the presence of a passive observer (Experiment 2). This pattern of results is compatible with the hypothesis that participants used a 
selective mechanism to stop when they performed the task together with a co-actor. However, the significant difference in SSRT obtained in Experiment 1 is not sufficient on its own as evidence that, at a neurocognitive level, two different mechanisms of stopping were activated (Aron \& Verbruggen, 2008). To obtain a direct measure of stopping selectivity in Experiment 3, we used TMS to probe the corticospinal excitability of a task-irrelevant leg muscle while participants performed a stop-signal task alone or alongside a co-actor. In line with predictions, results showed that task-irrelevant leg suppression was significantly reduced for the Joint condition compared to the Solo condition. When participants acted alone, stopping the hand had global suppressive effects across effectors not related to the task. When they performed the stop-signal task alongside a coactor, they used a more selective mechanism that allowed the suppression of a specific response tendency. In combination, the prolonged SSRT and the significant reduction in leg suppression provide strong evidence in favour of the recruitment of a more selective stopping mechanism in the Joint condition compared to the Solo condition.

\section{Selective joint stopping}

How was the selective stopping mechanism activated in the Joint condition?

Recent studies indicate that when people do not have foreknowledge about the particular response they may need to prevent, they use a fast-acting stopping mechanism that suppresses both the prepared response and other potential responses. However, when they have foreknowledge regarding which response they may have to stop, they use a more selective, but also slower mechanism (e.g. Claffey et al., 2010; Majid et al., 2012). This occurs, for instance, when they are cued in advance about which hand response to stop ("Maybe stop right" or "Maybe stop left"): participants represent two response alternatives and selectively prepare to stop a specific response (e.g. Aron \& Verbruggen, 2008). One possibility therefore is that in the joint task participants represented separate stimulus-response mappings for each agent, and selectively prepared to stop their own response. 
The ideomotor theory predicts that observing somebody perform an action should activate corresponding motor representations in the observer and create an action tendency (Greenwald, 1970; Jeannerod \& Frak, 1999; Prinz, 1997). During a joint stopping task, action possibilities at the other's disposal might thus become represented, influencing the mechanism used to stop one's own response. Participants may be slower to stop their response tendency because they use a more selective stopping mechanism to control their own action independently of what the co-actor is doing. If this is correct, then one would expect joint SSRT to correlate with SSRT as measured in a task requiring selective stopping. Moreover, joint stopping may be expected to be even more selective in social conditions that emphasize behavioral selectivity, e.g. when the participant and the co-actor have different task instructions. This prediction could be tested by using stopping paradigms which require one to withhold their action when it is the other's turn to act.

An alternative, yet not mutually exclusive, possibility is that participants engaged a more selective stopping mechanism when acting alongside the co-actor because the presence of the coactor acted as a reference frame for the spatial coding of their own action. In the cued selective stopping task participants are explicitly instructed to prepare to stop a particular hand ("Maybe Stop Right" or "Maybe Stop Left"; e.g. Aron \& Verbruggen, 2008). Similarly to this cueing of the possibility of stopping the left/right hand response, the co-actor may provide a spatial frame for referential response coding - just as one's own action alternatives provide a reference frame for relative response coding in two-choice tasks (Dolk et al., 2011; Dolk et al., 2013; Guagnano et al., 2010). For instance, when the co-actor's response occurs on the right side, the participant's response is coded as left. According to this referential-coding account, the key enabler of selective stopping in the Joint condition would thus be the encoding of the participant's own action with reference to the other person's action. The effect observed in the Joint condition would not be specific for social actions, but should be observed for both social action and non-social action events, as long as they provide a spatial reference frame which allows for referential coding. In line with this, Dolk and colleagues (2013) recently demonstrated that spatial compatibility effects such 
as the Social Simon effect do not necessarily require social nor movement features to occur, but can be elicited by any event salient enough to draw spatial attention. Studies modifying the social nature of the stop-task situation - in terms of both action and procedural characteristics - may help to clarify whether in the absence of foreknowledge cues, co-representation of a human co-actor's action is necessary for a transition from global to selective inhibition to occur.

\section{What are the neural mechanisms for joint stopping?}

Modulation of leg suppression in Experiment 3 indicates that stopping the hand had global suppressive effects when acting alone, but not when acting alongside a co-actor. When combined with previous evidence, we speculate that this finding argues in favour of two different neural mechanisms for stopping. Global stopping has been proposed to engage a hyperdirect neural pathway characterized by fast and direct projections from the cortex (i.e., right inferior frontal cortex and presupplementary motor area) to the subthalamic nucleus of the basal ganglia (Aron et al., 2007; Aron \& Poldrack 2006). The subthalamic nucleus is a deep brain structure with diffuse excitatory projections to output nuclei of the basal ganglia (including the globus pallidus pars interna), which, in turn, exert a diffuse inhibitory influence over M1 via the thalamus. Therefore, recruitment of the subthalamic nucleus could result in the rapid suppression of activity throughout the motor system.

In contrast to the proposed global stopping mechanism, selective stopping may instead be implemented via an indirect pathway from the striatum to the globus pallidus pars externa and then to the globus pallidus pars interna. The termination pattern of striatal neurons onto the globus pallidus pars interna, and from globus pallidus pars externa to the globus pallidus pars interna has a very focused effect and may therefore lead to suppression of specific representations in M1 (for a review of neural systems underlying motor stopping see Aron, 2011; Jahfari et al., 2012, 2011; Chikazoe, 2010; Chambers et al., 2009; Eagle et al., 2008). 
Cued stopping when participants have foreknowledge about which response they may need to stop has been proposed to target the motor system via the slower, indirect pathway. The current results provide further evidence for different modes of stopping, a global mechanism and a selective one, and suggest that a more selective pathway may be used not only when participants are cued in advance about which response to stop, but also when they act alongside another person. While future studies will be necessary to clarify whether joint stopping is implemented via the same fronto-striatal-pallidal pathway involved in cued stopping, these results provide novel insights into the circumstances which may activate global versus selective mechanisms in the stopping of action.

\section{Conclusions}

Acting together requires the recruitment of control processes which ensure joint stopping of action. Our results provide the first demonstration that the social context exerts a specific influence on the mechanisms underlying stopping. Participants use a fast global inhibition mechanism when acting alone; however, they recruit a more selective and slower stopping mechanism when acting with someone else. These findings motivate a richer model of how people control their inappropriate response tendencies and suggest that joint stopping and solo stopping recruit different suppression mechanisms.

\section{Acknowledgments}

This work received funding from the European Research Council under the European Union's Seventh Framework Programme (FP7/2007-2013) / ERC grant agreement n. 312919. C.C. is supported by the Economic and Social Research Council (ES/K00140X/1). Authors would like to thank Claudio Campus for the technical support. 


\section{Abbreviations}

ITI, intertrial interval; M1, primary motor cortex; MEP, motor evoked potential; OSP, optimal scalp position; $p(\mathrm{R} \mid \mathrm{S})$, probability of responding to a stop signal; $p(\mathrm{~S} \mid \mathrm{S})$, probability of stopping to a stop signal; rMT, resting motor threshold; RMS, root mean square; RT, reaction time; SSD, stop-signal delay; SSRT, stop-signal reaction time; TA, tibialis anterior; TMS, transcranial magnetic stimulation 


\section{References}

Aiello, J. R., \& Douthitt, E. A. (2001). Social facilitation from Triplett to electronic performance monitoring. Group Dynamics: Theory, Research, and Practice, 5, 163.

Aron, A. R. (2009). Introducing a special issue on stopping action and cognition. Neurosci. and Biobehav. Rev., 33, 611.

Aron, A. R. (2011). From reactive to proactive and selective control: developing a richer model for stopping inappropriate responses. Biol. Psychiatry, 69, e55-e68.

Aron, A. R., \& Poldrack, R. A. (2006). Cortical and subcortical contributions to Stop signal response inhibition: role of the subthalamic nucleus. J. Neurosci., 26, 2424-2433.

Aron, A. R., \& Verbruggen, F. (2008). Stop the Presses Dissociating a Selective From a Global Mechanism for Stopping. Psychol. Sci., 19, 1146-1153.

Aron, A. R., Durston, S., Eagle, D. M., Logan, G. D., Stinear, C. M., \& Stuphorn, V. (2007). Converging evidence for a fronto-basal-ganglia network for inhibitory control of action and cognition. J. Neurosci., 27, 11860-11864.

Badry, R., Mima, T., Aso, T., Nakatsuka, M., Abe, M., Fathi, D., et al. (2009). Suppression of human cortico-motoneuronal excitability during the Stop-signal task. Clin. Neurophysiol., 120, 1717-1723.

Band, G. P., Van Der Molen, M. W., \& Logan, G. D. (2003). Horse-race model simulations of the stop-signal procedure. Acta Psychol., 112, 105-142.

Bard, E. G., Sotillo, C., Anderson, A. H., Thompson, H. S., \& Taylor, M. M. (1996). The DCIEM map task corpus: spontaneous dialogue under sleep deprivation and drug treatment. Speech Commun. 20, 71-84.

Bond, C. F., \& Titus, L. J. (1983). Social facilitation: a meta analysis of 241 studies. Psychol. Bull. 94, 265-292.

Bratman, M. E. (1992). Shared cooperative activity. Philos. Rev., 101, 327-341. 
Cavallo, A., Becchio, C., Sartori, L., Bucchioni, G., \& Castiello, U. (2012). Grasping with tools: corticospinal excitability reflects observed hand movements. Cereb. Cortex, 22, 710-716.

Chambers, C. D., Garavan, H., \& Bellgrove, M. A. (2009). Insights into the neural basis of response inhibition from cognitive and clinical neuroscience. Neurosci. Biobehav. Rev., 33, 631.

Chikazoe, J. (2010). Localizing performance of go/no-go tasks to prefrontal cortical subregions. Curr. Opin. Psychiatry, 23, 267-272.

Claffey, M. P., Sheldon, S., Stinear, C. M., Verbruggen, F., \& Aron, A. R. (2010). Having a goal to stop action is associated with advance control of specific motor representations. Neuropsychologia, 48, 541-548.

Coxon, J. P., Stinear, C. M., \& Byblow, W. D. (2009). Stop and go: the neural basis of selective movement prevention. J. Cogn. Neurosci., 21, 1193-1203.

Dolk, T., Hommel, B., Colzato, L. S., Schütz-Bosbach, S., Prinz, W., \& Liepelt, R. (2011). How "social" is the social Simon effect?. Front. Psychol., 2.

Dolk, T., Hommel, B., Prinz, W., \& Liepelt, R. (2013). The (Not So) Social Simon Effect: A Referential Coding Account. J. Exp. Psychol. Hum. Percept. Perform., 39, 1248-1260.

Duque, J., \& Ivry, R. B. (2009). Role of corticospinal suppression during motor preparation. Cereb. Cortex, 19, 2013-2024.

Eagle, D. M., Baunez, C., Hutcheson, D. M., Lehmann, O., Shah, A. P., \& Robbins, T. W. (2008). Stop-signal reaction-time task performance: role of prefrontal cortex and subthalamic nucleus. Cereb. Cortex, 18, 178-188.

Greenwald, A. G. (1970). Sensory feedback mechanisms in performance control: with special reference to the ideo-motor mechanism. Psychol. Rev., 77, 73.

Guagnano, D., Rusconi, E., \& Umiltà, C. A. (2010). Sharing a task or sharing space? On the effect of the confederate in action coding in a detection task. Cognition, 114, 348-355.

Heed, T., Habets, B., Sebanz, N., \& Knoblich, G. (2010). Others’ actions reduce crossmodal integration in peripersonal space. Curr. Biol., 20, 1345-1349. 
Jeannerod, M., \& Frak, V. (1999). Mental imaging of motor activity in humans. Curr. Opin. Neurobiol., 9, 735-739.

Jahfari, S., Verbruggen, F., Frank, M. J., Waldorp, L. J., Colzato, L., Ridderinkhof, K. R., \& Forstmann, B. U. (2012). How preparation changes the need for top-down control of the basal ganglia when inhibiting premature actions. J. Neurosci., 32, 10870-10878.

Jahfari, S., Waldorp, L., van den Wildenberg, W. P., Scholte, H. S., Ridderinkhof, K. R., \& Forstmann, B. U. (2011). Effective connectivity reveals important roles for both the hyperdirect (fronto-subthalamic) and the indirect (fronto-striatal-pallidal) fronto-basal ganglia pathways during response inhibition. J. Neurosci., 31, 6891-6899.

Knoblich, G., Butterfill, S., \& Sebanz, N. (2011). Psychological research on joint action: theory and data. In Ross, B.H. (eds), The psychology of learning and motivation. The psychology of learning and theory: Advances in research and theory. Elsevier, San Diego, pp. 59-101.

Lappin, J. S., \& Eriksen, C. W. (1966). Use of a delayed signal to stop a visual reaction-time response. J. Exp. Psychol., 72, 805.

Levitt, H. (1971). Transformed up-down methods in psychoacoustics. J. Acoust. Soc. Am., 49, 467.

Logan, G. D. (1981). Attention, automaticity, and the ability to stop a speeded choice response. Attention Perform. IX, 205-222.

Logan, G. D. (1994). On the ability to inhibit thought and action: A users' guide to the stop signal paradigm. Inhibitory Processes in Attention, Memory and Language, 189-239.

Logan, G. D., \& Cowan, W. B. (1984). On the ability to inhibit thought and action: A theory of an act of control. Psychol. Rev., 91, 295-327.

Majid, D. A., Cai, W., George, J. S., Verbruggen, F., \& Aron, A. R. (2012). Transcranial magnetic stimulation reveals dissociable mechanisms for global versus selective corticomotor suppression underlying the stopping of action. Cereb. Cortex, 22, 363-371.

Ollman, R. T. (1973). Simple reactions with random countermanding of the "go" signal. Attention Perform., IV, 571-581. 
Prinz, W. (1997). Perception and action planning. Eur J Cogn Psychol, 9, 129-154.

Ramnani, N., \& Miall, R. C. (2004). A system in the human brain for predicting the actions of others. Nat. Neurosci., 7, 85-90.

Rossi, S., Hallett, M., Rossini, P. M., \& Pascual-Leone, A. (2009). Safety, ethical considerations, and application guidelines for the use of transcranial magnetic stimulation in clinical practice and research. Clin. Neurophysiol., 120, 2008-2039.

Rossini, P. M., Barker, A. T., Berardelli, A., Caramia, M. D., Caruso, G., Cracco, R. Q., et al. (1994). Noninvasive electrical and magnetic stimulation of the brain, spinal cord and roots: basic principles and procedures for routine clinical application. Report of an IFCN committee. Electroencephalogr. Clin. Neurophysiol, 91, 79-92.

Schuch, S., \& Tipper, S. P. (2007). On observing another person's actions: Influences of observed inhibition and errors. Percept. Psychophys., 69, 828-837.

Sebanz, N., Bekkering, H., \& Knoblich, G. (2006). Joint action: bodies and minds moving together. Trends Cogn Sci, 10, 70-76.

Sebanz, N., Knoblich, G., \& Prinz, W. (2003). Representing others' actions: Just like one's own?. Cognition, 88, B11-B21.

Sebanz, N., Knoblich, G., \& Prinz, W. (2005). How two share a task: corepresenting stimulusresponse mappings. J. Exp. Psychol. Hum. Percept. Perform, 31, 1234-1246.

Sebanz, N., Knoblich, G., Prinz, W., \& Wascher, E. (2006). Twin Peaks: An ERP study of action planning and control in co-acting individuals. J. Cogn. Neurosci., 18, 859-870.

Stinear, C. M., Coxon, J. P., \& Byblow, W. D. (2009). Primary motor cortex and movement prevention: where Stop meets Go. Neurosci. Biobehav. Rev., 33, 662-673.

Tsai, C.-C., Kuo, W.-J., Jing, J.-T., Hung, D. L., \& Tzeng, O. J.-L. (2006). A common coding framework in self-other interaction: Evidence from joint action task. Exp. Brain Res., 175, 353362. 
Verbruggen, F., \& Logan, G. D. (2009). Models of response inhibition in the stop-signal and stopchange paradigms. Neurosci. Biobehav. Rev., 33, 647-661.

Verbruggen, F., Chambers, C. D., \& Logan, G. D. (2013). Fictitious Inhibitory Differences How Skewness and Slowing Distort the Estimation of Stopping Latencies. Psychol. Sci., 24(3), 352362.

Verbruggen, F., Logan, G. D., \& Stevens, M. A. (2008). STOP-IT: Windows executable software for the stop-signal paradigm. Behav. Res. Methods, 40, 479-483.

Wassermann, E. M. (1998). Risk and safety of repetitive transcranial magnetic stimulation: report and suggested guidelines from the International Workshop on the Safety of Repetitive Transcranial Magnetic Stimulation, June 5-7, 1996. Electroencephalogr. Clin. Neurophysiol., 108, 1-16.

World Medical Association. (2008). World Medical Association Declaration of Helsinki: ethical principles for medical research involving human subjects. World Medical Association. 
Table 1. Experiment 1: comparison between Solo and Joint conditions

\begin{tabular}{lll} 
& \multicolumn{1}{c}{ Solo } & Joint \\
\hline Go trials accuracy $(\%)$ & $.983 \pm .022$ & $.976 \pm .022$ \\
Probability of stopping $\mathrm{p}(\mathrm{S} \mid \mathrm{S})$ & $.495 \pm .055$ & $.487 \pm .056$ \\
Go RT $(\mathrm{ms})$ & $508 \pm 79$ & $501 \pm 73$ \\
SSD $(\mathrm{ms})^{*}$ & $291 \pm 73$ & $251 \pm 60$ \\
SSRT $(\mathrm{ms})^{*}$ & $231 \pm 50$ & $267 \pm 59$ \\
\hline
\end{tabular}

Behavioural indices obtained from Experiment 1. All values represent mean \pm standard deviation. Asterisks indicate significant differences between conditions $(\mathrm{p}<.05)$

Table 2. Experiment 3: comparison between Solo and Joint conditions

\begin{tabular}{lcl} 
& Solo & Joint \\
\hline Go trials accuracy $(\%)$ & $.955 \pm .030$ & $.954 \pm .037$ \\
Probability of stopping $\mathrm{p}(\mathrm{S} \mid \mathrm{S})$ & $.524 \pm .060$ & $.515 \pm .049$ \\
Go RT (ms) & $420 \pm 72$ & $434 \pm 59$ \\
SSD (ms) & $157 \pm 69$ & $165 \pm 63$ \\
SSRT (ms)* & $279 \pm 52$ & $310 \pm 55$ \\
MEP Stop Ratio* & $.97 \pm .13$ & $1.05 \pm .11$ \\
\hline
\end{tabular}

Behavioural and EMG indices obtained from Experiment 3. All values represent mean \pm standard deviation. Asterisks indicate significant differences between conditions $(\mathrm{p}<.05)$ 


\section{Figure legends}

Figure 1. Example of experimental procedure for Experiment 3. Each trial began with a $500 \mathrm{~ms}$ fixation cross, followed by a $500 \mathrm{~ms}$ blank screen. The imperative Go signal was then presented. On Go trials (A) the Go signal remained on the screen until participants responded, or until $900 \mathrm{~ms}$ had elapsed. On Stop trials (B), following a variable SSD, a red cross Stop signal appeared over the circle/square indicating participants to withhold their responses and this remained on the monitor for the duration of the trial. The SSD was initially set at $250 \mathrm{~ms}$ and was then adjusted continuously with the staircase converging tracking procedure. The timing of the TMS pulse was calculated by subtracting $100 \mathrm{~ms}$ from mean Go RT obtained during the Practice session. 15 additional MEPs (TMS pulse, baseline) were recorded $300 \mathrm{~ms}$ before the onset of the Go signal for 10 Go trials and 5 Stop trials and served as a baseline.

Figure 2. (A) Peak-to-peak amplitude scores recorded from TA muscles during Solo and Joint conditions. MEP amplitudes are expressed as a Stop Ratio \pm s.e.m. calculated for each condition by dividing the mean MEP amplitude of the Stop trials by the mean MEP amplitude resulting from the Go trials. (B) Individual MEP Stop Ratio difference was calculated for each participant by subtracting the MEP Stop Ratio of the Joint condition from the MEP Stop Ratio of the Solo condition. 
Figure 1

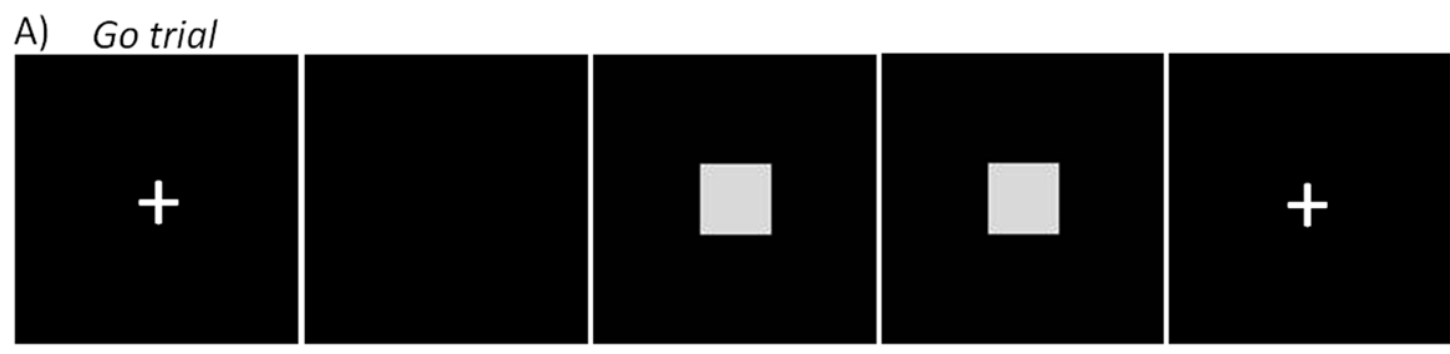

B) Stop trial

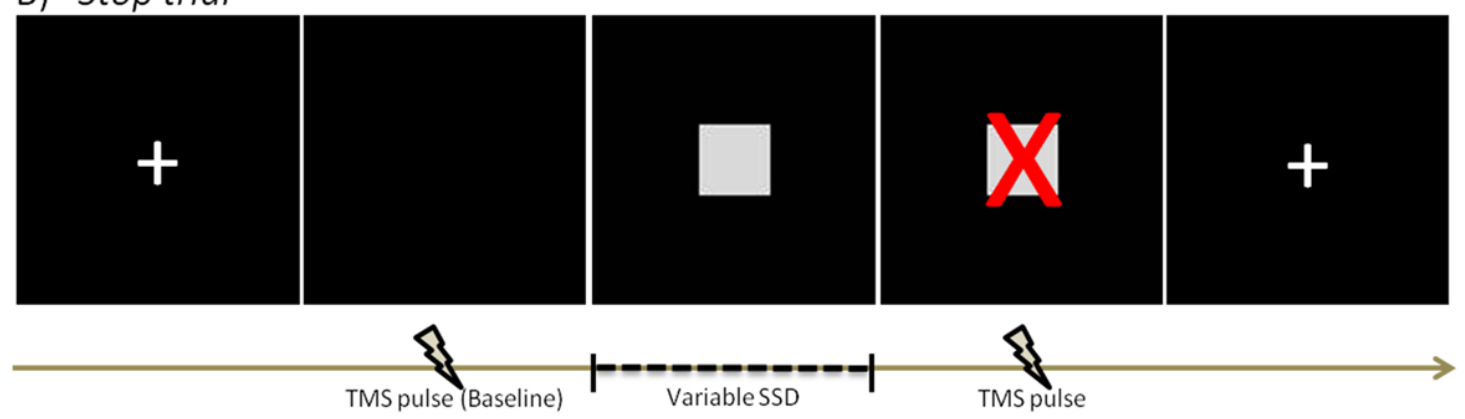


Figure 2
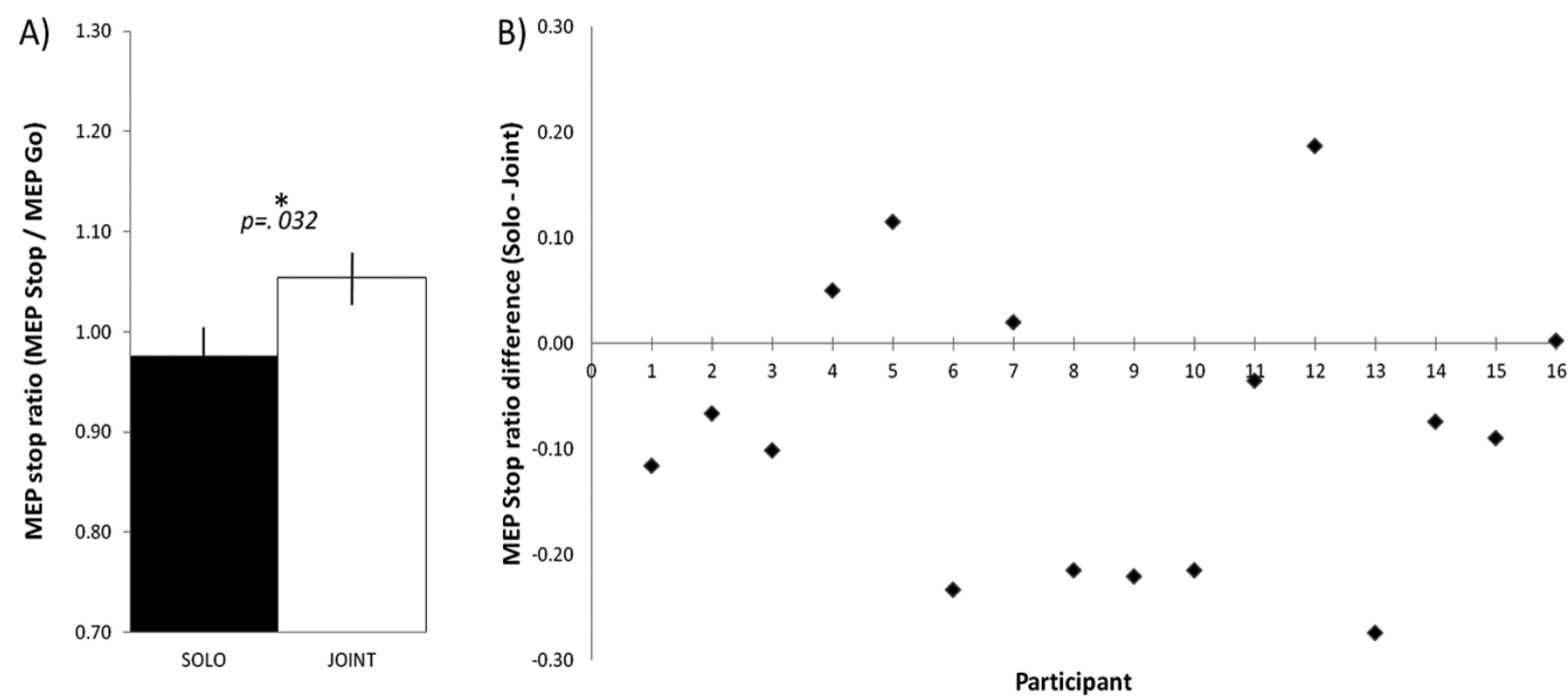